\title{
Super-Poincaré and Nash-type inequalities for Subordinated Semigroups
}

\author{
Ivan Gentil ${ }^{*}$ Patrick Maheux ${ }^{\dagger}$
}

August 9, 2018

\begin{abstract}
We prove that if a super-Poincaré inequality is satisfied by an infinitesimal generator $-A$ of a symmetric contracting semigroup then it implies a corresponding super-Poincaré inequality for $-g(A)$ with any Bernstein function $g$. We also study the converse statement. We deduce similar results for the Nash-type inequality. Our results applied to fractional powers of $A$ and to $\log (I+A)$ and thus generalize some results of [B-M] and [W1]. We provide several examples.
\end{abstract}

Key words: Super-Poincaré inequality, Nash-type inequality, Symmetric semigroup, Subordination in the sense of Bochner, Bernstein function, Super-Poincaré profile.

AMS 2010: 39B62, 47D60, 26A33, 46T12.

\section{Introduction and main results}

Let $\left(T_{t}\right)_{t>0}$ be a strongly continuous semigroup on $L^{2}(X, \mu)$ with $(X, \mu)$ a $\sigma$-finite measure space. We assume that, for each $t>0, T_{t}$ is a symmetric contraction on $L^{2}$. The infinitesimal generator of $\left(T_{t}\right)_{t>0}$ on $L^{2}$ denoted by $-A$ is a non-negative, closed and symmetric operator densely defined on $L^{2}$. We shall not distinguish $-A$ and its selfadjoint Friedrich extension. Moreover, we suppose that, for each $t>0, T_{t}$ is a contraction from $L^{1} \cap L^{2}$ to itself. So, for each $t>0$, the operator $T_{t}$ can be uniquely extended as a contraction on each $L^{p}, 1 \leq p \leq+\infty$. This extension $T_{t}^{(p)}$ will be again denoted by $T_{t}$. Recall that the infinitesimal generator $-A$ on $L^{2}(\mu)$ of $\left(T_{t}\right)_{t>0}$ is defined by

$$
-A f=\lim _{t \rightarrow 0^{+}} \frac{T_{t} f-f}{t} \in L^{2}
$$

on the domain $\mathcal{D}(A)$ which is the set of functions $f$ such that the limit just above exists in $L^{2}(\mu)$. We denote by $(.,$.$) the inner product on L^{2}$ and by $\|.\|_{p}$ the $L^{p}$-norm.

\footnotetext{
*Institut Camille Jordan, Université Claude Bernard Lyon 1. 43 boulevard du 11 novembre 1918. 69622 Villeurbanne cedex. France. gentil@math.univ-lyon1.fr

${ }^{\dagger}$ Fédération Denis Poisson, Département de Mathématiques, Université d'Orléans. Rue de Chartres. B.P. 6759 - 45067 Orléans cedex 2. France.patrick.maheux@univ-orleans.fr
} 
We shall say that $A$ satisfies a super-Poincaré inequality with rate function $\beta:(0,+\infty) \rightarrow$ $(0,+\infty)$ if, for any $f \in \mathcal{D}(A) \cap L^{1}(\mu)$,

$$
\|f\|_{2}^{2} \leq r(A f, f)+\beta(r)\|f\|_{1}^{2}, r>0 .
$$

More generally, we say that $A$ satisfies a $\left(r_{0}, r_{1}\right)$-super-Poincaré inequality if $(1)$ holds for $r \in\left(r_{0}, r_{1}\right)$ with $0<r_{0}<r_{1} \leq+\infty$. See examples below Theorem 1.2. Note that we can always assume that $\beta$ is non-increasing by considering what we shall call the super-Poincaré profile $\beta_{p}(\leq \beta)$ of $A$ defined, for any $r>0$, by

$$
\beta_{p}(r):=\sup \left\{\|f\|_{2}^{2}-r(A f, f): f \in \mathcal{D}(A) \cap L^{1}(\mu),\|f\|_{1} \leq 1\right\} .
$$

We shall say that $A$ satisfies a Nash-type inequality with non-decreasing rate function $D:[0,+\infty) \rightarrow[0,+\infty)$ if, for any $f \in \mathcal{D}(A) \cap L^{1}(\mu)$,

$$
\|f\|_{2}^{2} D\left(\|f\|_{2}^{2}\right) \leq(A f, f), \quad\|f\|_{1} \leq 1 .
$$

$D$ is then a Nash function for $A$. It is well known that the inequalities (1) and (3) are essentially equivalent, see Proposition 2.2.

We now briefly recall some definitions and some facts about the subordination of semigroups in the sense of Bochner.

A Bernstein function $g$ is a $\mathcal{C}^{\infty}$ function $g:(0,+\infty) \rightarrow(0,+\infty)$ satisfying

$$
(-1)^{n-1} g^{(n)}(s) \geqslant 0
$$

for any $n \in \mathbb{N}^{*}, s>0$. There exists a convolution semigroup of sub-probability measures $\left(\nu_{t}^{g}\right)_{t>0}$ on $(0,+\infty)$ with density $\left(\eta_{t}^{g}\right)_{t>0}$ with respect to the Lebesgue measure $d s$ such that the Laplace transform of $\nu_{t}^{g}$ is given by

$$
\int_{0}^{+\infty} e^{-s x} d \nu_{t}^{g}(s)=\int_{0}^{+\infty} e^{-s x} \eta_{t}^{g}(s) d s=e^{-t g(x)}, x>0 .
$$

There is one-to-one correspondence between $g$ and $\left(\eta_{t}^{g}\right)_{t>0}$, see [J] p.177, [B-F].

Let recall that all Bernstein functions $g$ can be written by Lévy-Khintchine formula

$$
g(x)=a+b x+\int_{0}^{\infty}\left(1-e^{-\lambda x}\right) d \nu(\lambda)=a+b x+J(x)
$$

with $a, b \geqslant 0$ and $\nu$ a positive measure on $(0,+\infty)$ such that $\int_{0}^{\infty} \frac{\lambda}{1+\lambda} d \nu(\lambda)<\infty$. The triplet $(a, b, \nu)$ is uniquely determined by $g$, see Theorem 3.9.4 in [J] p.174. We have $a=g(0)=0$ if and only if $\nu_{t}^{g}$ is a probability measure for any $t>0$. For instance with $a=b=0$, the Lévy measure associated to $g(x)=x^{\alpha}$ with $\alpha \in(0,1)$ (resp. $g(x)=$ $\ln (1+x))$ is given by $d \nu(\lambda)=\frac{\alpha}{\Gamma(1-\alpha)} \lambda^{-1-\alpha} d \lambda\left(\right.$ resp. $\left.d \nu(\lambda)=\frac{e^{-\lambda}}{\lambda} d \lambda\right)$.

Now, let $\left(E_{\lambda}\right)_{\lambda \in[0,+\infty)}$ be the spectral resolution of the non-negative self-adjoint operator $A$ and $\Psi:[0,+\infty) \longrightarrow \mathbb{R}$ any measurable function. The operator $\Psi(A)$ is defined on $L^{2}(\mu)$ by the formula

$$
\Psi(A) f=\int_{0}^{+\infty} \Psi(\lambda) d E(\lambda) f
$$

with domain

$$
\mathcal{D}(\Psi(A))=\left\{f \in L^{2}: \int_{0}^{+\infty}|\Psi(\lambda)|^{2} d(E(\lambda) f, f)<\infty\right\}
$$


We shall set $(\Psi(A) f, f)=+\infty$ when $f \notin \mathcal{D}(\Psi(A))$. When $\Psi$ has real non-negative values, the operator $\Psi(A)$ is non-negative and self-adjoint on $L^{2}(\mu)$. It defines a symmetric semigroup of contractions on $L^{2}$ by the spectral formula:

$$
e^{-t \Psi(A)} f=\int_{0}^{+\infty} e^{-t \Psi(\lambda)} d E(\lambda) f, \quad f \in L^{2}(\mu) .
$$

The operator $e^{-t \Psi(A)}$ will be denoted by $T_{t}^{\Psi}$. When $\Psi=g$ is a Bernstein function, it can be easily shown that the semigroup $\left(T_{t}^{g}\right)_{t>0}$ satisfies also the so-called subordination formula

$$
T_{t}^{g}=\int_{0}^{+\infty} T_{s} d \nu_{t}^{g}(s)=\int_{0}^{+\infty} T_{s} \eta_{t}^{g}(s) d s
$$

Let $t>0$, since $T_{t}$ is a contraction on $L^{p}$ (resp. positive or sub-markovian on $L^{2}$ ) then $T_{t}^{g}$ is a contraction on $L^{p}$ (resp. positive or sub-markovian on $L^{2}$ ).

Among many examples of Bernstein functions, we are interested at least in the following ones:

i. The fractional subordinator (one-sided $\alpha$-stable process): $g(x)=x^{\alpha}, x>0,(0<$ $\alpha<1)$. Then $g(A)=A^{\alpha}$.

ii. The Gamma subordinator: $g(x)=\log (1+x)$. Then $g(A)=\log (I+A)$ where $I$ denotes the identity operator on $L^{2}(\mu)$.

iii. The generalization of the previous example: $g(x)=\left[\log \left(1+x^{\alpha}\right)\right]^{\gamma}$ with $0<\alpha<1$ and $0<\gamma \leq 1$. It gives $g(A)=\left[\log \left(I+A^{\alpha}\right)\right]^{\gamma}$. When $\gamma=1, g$ is called the geometric $\alpha$-stable subordinator, see [S-S-V].

iv. Elementary functions $g_{\lambda}(x)=1-e^{-\lambda x}, \lambda>0$. Then $g_{\lambda}(A)=I-T_{\lambda}$.

For a recent study of the case (ii), see [S-S-V]. See also [Sc-S-V] Chap.15 for a long list of examples of Bernstein functions.

We have the inclusion $\mathcal{D}(A) \subset \mathcal{D}(g(A))$ and by formula (5), for any $f \in \mathcal{D}(A)$,

$$
g(A) f=a f+b A f+\int_{0}^{\infty}\left(f-T_{\lambda} f\right) d \nu(\lambda) .
$$

See $[\mathrm{Sc}-\mathrm{S}-\mathrm{V}]$ Example 11.6 (note that our $A$ is their $-A$ ).

Throughout all the paper, we shall always assume implicitly that the functions $f$ are in the domain of the operator under consideration. If not, we set $(g(A) f, f)=+\infty$.

We now state the main results of the paper.

Theorem 1.1 Let $-A$ be an infinitesimal generator of a semigroup as above and $g$ be a Bernstein function. If $-A$ satisfies a super-Poincaré inequality with rate function $\beta$.

Then the infinitesimal generator $-g(A)$ satisfies a $\left(r_{0}, r_{1}\right)$-super-Poincaré inequality with rate function $\beta_{g}(r)=\beta\left(\frac{1}{g^{-1}(1 / r)}\right), r \in\left(r_{0}, r_{1}\right)$ where $r_{0}=\frac{1}{g(+\infty)}$ and $r_{1}=\frac{1}{g\left(0^{+}\right)}$.

Note that by (5), $g$ is either strictly increasing and $g^{-1}$ is defined from $\left(g\left(0^{+}\right), g(+\infty)\right)$ into $(0,+\infty)$ or $g$ is constant and $\left(r_{0}, r_{1}\right)$ is empty. The Bernstein function $g$ is bounded if and only if $b=0$ and $\nu$ is a bounded measure, see [J] p.174. If $g$ is not bounded, 
i.e. $g(+\infty)=+\infty$, then we have $r_{0}=0$. If $g\left(0^{+}\right)=a=0$ then $r_{1}=+\infty$. But if $a=g\left(0^{+}\right)>0$ then we have the obvious spectral gap inequality

$$
\|f\|_{2}^{2} \leq \frac{1}{a}(g(A) f, f) .
$$

In other words,

$$
\|f\|_{2}^{2} \leq r(g(A) f, f)+\beta_{g}(r)\|f\|_{1}^{2} \text { with } \beta_{g}(r)=0, r \in[1 / a,+\infty) .
$$

So, in any case we can consider that $r_{1}=+\infty$.

In particular, Theorem 1.1 applies to several important examples. We provide a short list of couples $\left(g(A), \beta_{g}\right)$ just below.

i. Fractional powers. If $g(A)=A^{\alpha}$ with $0<\alpha<1$ then $\beta_{\alpha}(r)=\beta\left(r^{\frac{1}{\alpha}}\right), r>0$, (improving constants given in [W1]).

ii. Gamma subordinator. If $g(A)=\log (I+A)$ then $\beta_{\log }(r)=\beta\left(\left(e^{1 / r}-1\right)^{-1}\right), r>0$.

iii. Generalized geometric stable subordinators. If $g(A)=\left[\log \left(I+A^{\alpha}\right)\right]^{\gamma}$ with $0<\alpha$, $\gamma \leq 1$, then

$$
\beta_{g}(r)=\beta\left(\left[e^{\left(\frac{1}{r}\right)^{\frac{1}{\gamma}}}-1\right]^{\frac{-1}{\alpha}}\right), r>0 .
$$

iv. Random walks. If $g(A)=I-T_{\lambda}=I-e^{-\lambda A}$ with $\lambda>0$ then

$$
\beta^{(\lambda)}(r)=\beta\left(\frac{\lambda}{\log \left(1+\frac{1}{r-1}\right)}\right), r>1 .
$$

The same super-Poincaré inequality for the generator $B_{\lambda}=I-T_{\lambda}$ with $\lambda>0$ can also be deduced by a different route, see (iii) of Proposition 2.1 below.

The second main result is similar with Nash-type inequality assumption.

Theorem 1.2 Let $-A$ be an infinitesimal generator of a semigroup as above satisfying a Nash-type inequality with rate function D. Set for $r>0$,

$$
\beta(r)=\sup _{x>0}(x-r x D(x)),
$$

and assume that $\beta(r)$ is finite for any $r>0$. Let $g$ be a Bernstein function.

$i$. Then $g(A)$ satisfies a Nash-type inequality of the form

$$
\|f\|_{2}^{2} D_{g, \beta}\left(\|f\|_{2}^{2}\right) \leq(g(A) f, f), \quad\|f\|_{1} \leq 1,
$$

where $D_{g, \beta}(x)=\sup _{r \in\left(r_{0}, r_{1}\right)}\left(\frac{1}{r}-\frac{1}{r x} \beta\left(\frac{1}{g^{-1}\left(\frac{1}{r}\right)}\right)\right), x>0$ and $\left(r_{0}, r_{1}\right)=\left(\frac{1}{g(+\infty)}, \frac{1}{g\left(0^{+}\right)}\right)$.

ii. Moreover, assume that $g$ is a bijection from $[0,+\infty)$ to itself and $\beta:(0,+\infty) \rightarrow$ $(0,+\infty)$ is a decreasing differentiable bijection. Then we have

$$
\|f\|_{2}^{2} \sup _{\rho>1}\left(1-\rho^{-1}\right)(g \circ D)\left(\rho^{-1}\|f\|_{2}^{2}\right) \leq(g(A) f, f), \quad\|f\|_{1} \leq 1,
$$

and, for any $x>0$,

$$
\sup _{\rho>1}\left(1-\rho^{-1}\right)(g \circ D)\left(\rho^{-1} x\right) \leq D_{g, \beta}(x) \leq g \circ D(x) .
$$


See Section 8 for the links between $D$ and $\beta$.

Our approach simplifies and generalizes the proofs of the main results of [B-M] and [W1]. The inequality (9) also clarifies the constants obtained in [B-M] for the fractional powers $A^{\alpha}$. With the same arguments of proof, we can replace $\|f\|_{1}$ in Theorems 1.1 and 1.2 by any non-negative functional $\Phi(f)$ satisfying $\Phi\left(T_{t} f\right) \leq \Phi(f), t>0$. Our results can be generalized in the same way in Hilbert spaces as in Wang's paper [W1]. But we shall not give details.

The paper is organized as follows:

In Section 1, we describe the setting of our study and we state the main theorems. In Section 2, we prove the main theorems of Section 1. More precisely: In Section 2.1, we first recall that the super-Poincaré inequality for $A$ is equivalent to the decay for the corresponding semigroup $\left(T_{t}\right)_{t>0}$. Sections 2.2 and 2.3 are devoted respectively to the proof of Theorem 1.1 and 1.2 for $g(A)$ with $g$ a Bernstein function using results of Section 2.1.

In Section 3, we briefly apply our results to study the eventual ultracontractivity property of subordinated semigroups. In Section 4, we provide several examples of settings where our results apply: 1) the Laplacian on the Euclidean space, 2) the Laplace-Beltrami operator on some complete Riemannian manifolds, 3) some hypoelliptic operators on Lie groups and 4) the Ornstein-Uhlenbeck operator on the Euclidean space.

In Section 5, we study the same results as in Theorem 1.1 and 1.2 concerning Nashtype and super-Poincaré inequalities but for $\Psi(A)$ with $\Psi$ convex. For these two type of inequalities, we use spectral representation of the generator. From these results, we deduce converse implications of Theorem 1.1 and 1.2 by noting that the inverse of a Bernstein function is a convex function.

In Section 6, we revisit the spectral gap in $L^{p}(\mu)$ for $g(A)$ using the approach by subordination as in Theorem 1.1.

In Section 7, we prove super-Poincaré inequalities for $g(\Delta)$ with $\Delta$ the Laplacian on $\mathbb{R}^{n}$ for a larger class of functions than Bernstein functions. Our tool is Fourier analysis as used in the original paper by J.Nash [N]. The afferent Nash-type inequalities can be deduced. The results are similar to Theorem 1.1 and 1.2 .

We conclude this paper by an Appendix Section 8. It concerns the Legendre transform which is underlying in the equivalence between Nash-type inequalities and super-Poincaré inequalities through the functions $\beta$ and $D$. We weaken the usual conditions on $\beta$ and $D$ of the N-functions theory, see [R-R] p.13, more adapted to our situation. We provide examples used in Section 4.

\section{Proof of main Theorems}

\subsection{Super-Poincaré inequality versus semigroup decay and Nash-type inequality}

We recall some known result used in the proof of Theorem 1.1 and 1.2.

For the proof of Theorem 1.1, we use in a crucial way the following result of F-Y. Wang namely the equivalence between Super-Poincaré inequality for $A$ and the exponential decay 
of the associated semigroup $\left(T_{t}\right)_{t>0}$, see [W2] p.230 or [W4] Lemma 3.3.5, see also [W3] p.3 and [W4] p.50 for extended results. This is the analogue of the equivalence between the usual exponential decay of a semigroup and Poincaré inequality. We recall the proof of this proposition for completeness and, additionally, we show (iii) that is the exponential decay of $T_{t}$ turns out to be the super-Poincaré inequality for the operator $I-T_{t}$. This operator is related to the elementary Bernstein function $g_{t}(x)=1-e^{-t x}$.

Proposition 2.1 Let $\left(T_{t}\right)_{t>0}$ be a semigroup as in Section 1 with infinitesimal generator $-A$ and let $\beta:(0,+\infty) \rightarrow(0,+\infty)$. Then the three following inequalities are equivalent:

$i$. For any $f \in \mathcal{D}(A) \cap L^{1}(\mu)$ and $r>0$,

$$
\|f\|_{2}^{2} \leq r(A f, f)+\beta(r)\|f\|_{1}^{2} .
$$

ii. For any $f \in L^{2}(\mu) \cap L^{1}(\mu), t>0$ and $r>0$,

$$
\left\|T_{t} f\right\|_{2}^{2} \leq e^{-2 t / r}\|f\|_{2}^{2}+\left(1-e^{-2 t / r}\right) \beta(r)\|f\|_{1}^{2} .
$$

iii. For any $f \in L^{2}(\mu) \cap L^{1}(\mu), t>0$ and $r>1$,

$$
\|f\|_{2}^{2} \leq r\left(\left(I-T_{t}\right) f, f\right)+\beta\left(\frac{t}{\log \left(1+\frac{1}{r-1}\right)}\right)\|f\|_{1}^{2} .
$$

The exponential $e^{-2 t / r}$ in (11) is suitable to deal with Laplace transforms (4) and this is the key point of our paper. This allows us to transfer easily (11) from $A$ to $g(A)$.

During the proof, we can notice that the equivalence between (10) and (11) holds for any fixed $a=r>0$ and fixed $b=\beta(r) \geqslant 0$. In particular, if (10) holds on some interval $\left(r_{0}, r_{1}\right)$ then (11) also holds on the same interval $\left(r_{0}, r_{1}\right)$ and conversely.

The inequality (12) corresponds exactly to a super-Poincaré inequality for $g_{\lambda}(x)=$ $1-e^{-\lambda x}(t=\lambda)$ for any $\lambda>0$. The equivalence between (10) and (12) is particularly interesting in terms of relationships between the super-Poincaré profile for generators $A$ and $B_{\lambda}=I-T_{\lambda}$ for any fixed $\lambda>0$, see (2) above for the definition of the profile. If $\beta_{p}(s), s>0$ is the super-Poincaré profile of $A$ and $\gamma_{p}^{(\lambda)}(r), r>1$ is the super-Poincaré profile of $B_{\lambda}$ then they correspond by the formulas

$$
\gamma_{p}^{(\lambda)}(r)=\beta_{p}\left(\frac{\lambda}{\log \left(1+\frac{1}{r-1}\right)}\right), r>1,
$$

or equivalently

$$
\beta_{p}(s)=\gamma_{p}^{(\lambda)}\left(1+\left(e^{\lambda / s}-1\right)^{-1}\right), s>0 .
$$

For instance in the Euclidean setting, the optimal Nash inequality (16) below provides the super-Poincaré profile for the Laplacian $\Delta$, namely $\beta_{p}(s)=C_{n} s^{-n / 2}$, for some optimal constant $C_{n}$. Thus, the super-Poincaré profile of $B_{\lambda}$ is explicit and given by

$$
\gamma_{p}^{(\lambda)}(r)=C_{n} \lambda^{-n / 2}\left(\log \left[1+\frac{1}{r-1}\right]\right)^{n / 2}, r>1 .
$$

We have the following interpretation in terms of random walks. For fixed $\lambda>0$, the kernel $h_{\lambda}$ of the operator $T_{\lambda}$ can be seen as a probability transition of a discrete random 
walk $\left(X_{k}\right)_{k}$ on $\mathbb{R}^{n}$ given by $\mathbb{P}\left(X_{k+1}=x, X_{k}=y\right)=h_{\lambda}(x-y)=\frac{1}{(4 \pi \lambda)^{n / 2}} \exp \left(-\frac{|x-y|^{2}}{4 \lambda}\right)$, $x, y \in \mathbb{R}^{n}$ and $k \in \mathbb{N} \cup\{0\}$. The operator $B_{\lambda}=I-T_{\lambda}$ is the generator of the continuoustime Markov semigroup $Q_{t}^{(\lambda)}=e^{-t B_{\lambda}}=e^{-t} \sum_{k \geqslant 0} \frac{t^{k}}{k !} T_{k \lambda}$ obtained by convolution with the following probability transition

$$
q_{t}^{(\lambda)}=e^{-t} \sum_{k \geqslant 0} \frac{t^{k}}{k !} h_{k \lambda}
$$

where $h_{0}=\delta_{0}\left(\delta_{a}\right.$ is the Dirac mass at $\left.a \in[0,+\infty)\right)$. This semigroup $Q_{t}^{(\lambda)}$ is subordinated to the heat semigroup $e^{-t \Delta}$ by the Poisson semigroup with jumps of size $\lambda$ defined on $[0,+\infty)$ by $\nu_{t}=\sum_{k \geqslant 0} \frac{t^{k}}{k !} e^{-t} \delta_{k \lambda}$ in (6), see [J] p.180.

Proof: Equivalence between (i) and (ii). Let $H(t)=e^{2 t / r}\left\|T_{t} f\right\|_{2}^{2}$ for $t>0$, fixed $r>0$ and $f \in \mathcal{D}(A) \cap L^{1}(\mu)$. We have

$$
H(t)-H(0)=\int_{0}^{t} H^{\prime}(u) d u=\int_{0}^{t} 2 e^{2 u / r}\left(\frac{1}{r}\left\|T_{u} f\right\|_{2}^{2}-\left(A T_{u} f, T_{u} f\right)\right) d u .
$$

By applying (10) to $T_{u} f$ and since $T_{u}$ is a contraction on $L^{1}(\mu)$, we deduce

$$
H(t)-H(0) \leq \frac{2}{r} \beta(r)\|f\|_{1}^{2}\left(\int_{0}^{t} e^{2 u / r} d u\right) .
$$

This proves (11) for $f \in \mathcal{D}(A) \cap L^{1}(\mu)$. For the general case, let $f \in L^{2}(\mu) \cap L^{1}(\mu)$ then there exists $f_{u}(u>0)$ such that $f_{u} \in \mathcal{D}(A)$ and $f_{u}$ converges to $f$ in $L^{1}(\mu)$ and $L^{2}(\mu)$ as $u \rightarrow 0^{+}$(e.g. $f_{u}=\frac{1}{u} \int_{0}^{u} T_{s} f d s$ ).

Conversely, let $r>0$ be fixed and $f \in \mathcal{D}(A) \cap L^{1}(\mu)$. The inequality (11) can be rewritten as

$$
\frac{\left\|T_{t} f\right\|_{2}^{2}-\|f\|_{2}^{2}}{2 t} \leq\left(\frac{e^{-2 t / r}-1}{2 t}\right)\|f\|_{2}^{2}+\beta(r)\|f\|_{1}^{2}\left(\frac{1-e^{-2 t / r}}{2 t}\right) .
$$

We conclude (10) by taking the limit as $t$ goes to 0 .

Equivalence between (ii) and (iii). Assume that (ii) holds, i.e.

$$
\left(T_{t} f, f\right)=\left\|T_{t / 2} f\right\|_{2}^{2} \leq e^{-t / r}\|f\|_{2}^{2}+\left(1-e^{-t / r}\right) \beta(r)\|f\|_{1}^{2}
$$

for any $t, r>0$. It is equivalent to

$$
\left(1-e^{-t / r}\right)\|f\|_{2}^{2} \leq\left(f-T_{t} f, f\right)+\left(1-e^{-t / r}\right) \beta(r)\|f\|_{1}^{2} .
$$

Let $g(x)=g_{t}(x)=1-e^{-t x}$. The last inequality reads as

$$
\|f\|_{2}^{2} \leq \frac{1}{g_{t}(1 / r)}\left(g_{t}(A) f, f\right)+\beta(r)\|f\|_{1}^{2} .
$$

Fix $t>0$. Let $\rho>1$ and choose $r>0$ such that $\rho=\frac{1}{g_{t}(1 / r)}$, i.e. $r=\frac{1}{g_{t}^{-1}(1 / \rho)}=\frac{t}{\log \left(1+\frac{1}{\rho-1}\right)}$. This yields $(1, \infty)$-super-Poincaré $(12)$ for the operator $I-T_{t}$ as expected. The converse is clear.

Now we recall that super-Poincaré and Nash-type inequalities are essentially equivalent under natural conditions on $\beta$ in (1) and on $D$ in (3). This result is more or less well known but we formulate the relations between $\beta$ and $D$ implicitly in terms of Legendre transforms, see Appendix Section 8 for a detailed discussion. 
Proposition 2.2 Let $A$ be a non-negative symmetric operator on $L^{2}(\mu)$.

i. Assume A satisfies a super-Poincaré inequality with rate function $\beta$ then it satisfies a Nash-type inequality with rate function

$$
D(x)=\sup _{t>0}\left(t-\frac{t \beta(1 / t)}{x}\right) \in(-\infty,+\infty], x>0 .
$$

The function $D$ is non-decreasing, finite on the set $(0, \sup \mathcal{G})$ where $\mathcal{G}=\left\{\|f\|_{2}^{2}, f \in\right.$ $\left.\mathcal{D}(A) \cap L^{1}(\mu),\|f\|_{1} \leq 1\right\}$ and $D(+\infty)=+\infty$.

ii. Conversely, suppose Nash-type inequality holds true for $A$ and a rate function $D$ : $[0,+\infty) \rightarrow \mathbb{R}$. Set

$$
\beta(r)=\sup _{x>0}(x-r x D(x)) \in(-\infty,+\infty] .
$$

Then A satisfies a super-Poincaré inequality with rate function $\beta$.

This proposition is in the spirit of Theorem 3.1 and Section 5 of [W2], see also Proposition 3.3.16 of [W4]. A much closer formulation can be found in [Bi-M], Proposition 2.1.

Note that if $\mathcal{G}$ of Proposition 2.2 is unbounded above as a subset of $\mathbb{R}$ then $D$ is finite in $(0, \infty)$. For many examples, $\beta$ is a non-negative non-increasing function and satisfies $\beta\left(0^{+}\right)=+\infty, \lim _{t \rightarrow 0^{+}} t \beta(1 / t)=0$ which implies that $D(x)$ is finite for any $x>0$, non-negative, continuous and non-decreasing, see Appendix Section 8 for details. Note also that we can always consider in (3) that $D$ is non-negative by replacing $D$ by $D_{+}=\sup (0, D)$ since $A$ is a non-negative operator.

Remark 2.3 i. Usually Nash-type inequality is written in the following form

$$
\Theta\left(\|f\|_{2}^{2}\right) \leq(A f, f), \quad\|f\|_{1} \leq 1 .
$$

But the equivalent expression (3) is more appropriate to deal with the Bernstein functions $g(x)=x^{\alpha}, \alpha \in(0,1)$ as shown in [B-M] and more generally for any Bernstein functions by Theorem 1.2 above.

ii. In the second statement, the assumption on the functional $(A f, f)$ can certainly be relaxed because only the existence of the function $\beta$ (which depends on this functional) is crucial for the proof.

Proof: (i) Assume that a super-Poincaré inequality holds true. For any $\|f\|_{1} \leq 1, f \neq 0$, $f \in \mathcal{D}(A)$ and any $r>0$, we easily deduce

$$
\|f\|_{2}^{2}\left(\frac{1}{r}-\frac{\beta(r)}{r\|f\|_{2}^{2}}\right) \leq(A f, f) .
$$

Taking the supremum over $r>0$, we get a Nash-type inequality with rate function $D$. Note that $D$ is automatically finite on the subset $\mathcal{G} \backslash\{0\}$ of $\mathbb{R}$ since in that case $(A f, f)<+\infty$ for any $f \in \mathcal{D}(A)$. On one hand, the set $\mathcal{G}$ is not empty since it contains 0 . On the other hand, if $f \in \mathcal{G}$ and $\lambda \in(0,1)$ then $\lambda f \in \mathcal{G}$. Hence $(0, \sup \mathcal{G}) \subset \mathcal{G}$ and $D$ is finite on $(0, \sup \mathcal{G})$. It is easily proved that $D$ is non-decreasing using the fact that $\beta>0$. Moreover, for any $x, t>0$, we have

$$
D(x) \geqslant t-\frac{t \beta(1 / t)}{x} .
$$


Therefore, $\liminf _{x \rightarrow+\infty} D(x) \geqslant t$ for any $t>0$. It implies $\lim _{x \rightarrow+\infty} D(x)=+\infty$.

(ii) By definition of $\beta$, one has for any $x, r>0$,

$$
\frac{x}{r}-\frac{\beta(r)}{r} \leq x D(x) .
$$

Let $x=\|f\|_{2}^{2}($ with $f \neq 0)$. So, for fixed $r>0$,

$$
\frac{\|f\|_{2}^{2}}{r}-\frac{1}{r} \beta(r) \leq\|f\|_{2}^{2} D\left(\|f\|_{2}^{2}\right) \text {. }
$$

By the Nash-type inequality, the last term is bounded by $(A f, f)$ when $\|f\|_{1} \leq 1$. Hence,

$$
\frac{\|f\|_{2}^{2}}{r}-\frac{1}{r} \beta(r) \leq(A f, f)
$$

which is super-Poincaré inequality. This concludes the proof of the proposition.

Note that when $\beta(r)=+\infty$ for some $r>0$ then super-Poincaré inequality is satisfied and the proof is also valid. For properties of $\beta$ defined by (13), see Appendix Section 8 .

\subsection{Proof of Theorem 1.1}

We suppose that $g$ is non-constant (if not there is nothing to prove since $\left(r_{0}, r_{1}\right)=\emptyset$ ). Assume that a super-Poincaré inequality holds true with rate function $\beta$. By Proposition 2.1, the inequality (11) is satisfied. Now by symmetry and semigroup property of $\left(T_{t}\right)_{t>0}$, this inequality (11) can be written as

$$
\left\|T_{t} f\right\|_{2}^{2}=\left(T_{2 t} f, f\right) \leq e^{-2 t / r}\|f\|_{2}^{2}+\beta(r)\|f\|_{1}^{2}\left(1-e^{-2 t / r}\right), t>0 .
$$

Let $s>0$ and set $t=s / 2$. We deduce for any $r>0$ and $s>0$,

$$
\left(T_{s} f, f\right) \leq e^{-s / r}\|f\|_{2}^{2}+\beta(r)\|f\|_{1}^{2}\left(1-e^{-s / r}\right) .
$$

By the subordination formula (6) and Fubini, we get for any $t, r>0, f \in L^{1}(\mu) \cap L^{2}(\mu)$ and any Bernstein function $g$,

$$
\begin{aligned}
\left(T_{t}^{g} f, f\right)= & \int_{0}^{+\infty}\left(T_{s} f, f\right) d \nu_{t}^{g}(s) \leq\left(\int_{0}^{+\infty} e^{-s / r} d \nu_{t}^{g}(s)\right)\|f\|_{2}^{2} \\
& +\beta(r)\|f\|_{1}^{2}\left(\int_{0}^{+\infty}\left(1-e^{-s / r}\right) d \nu_{t}^{g}(s)\right) .
\end{aligned}
$$

By the Laplace transform of the sub-probability $\nu_{t}^{g}$, we get

$$
\left(T_{t}^{g} f, f\right) \leq e^{-t g(1 / r)}\|f\|_{2}^{2}+\beta(r)\|f\|_{1}^{2}\left(1-e^{-t g(1 / r)}\right), \quad t, r>0 .
$$

Changing $t$ by $2 t$ and using symmetry and semigroup properties of $\left(T_{t}^{g}\right)$, we obtain

$$
\left\|T_{t}^{g} f\right\|_{2}^{2} \leq e^{-2 t g(1 / r)}\|f\|_{2}^{2}+\beta(r)\|f\|_{1}^{2}\left(1-e^{-2 t g(1 / r)}\right), \quad t, r>0 .
$$

Now let $\rho \in\left(r_{0}, r_{1}\right):=\left(\frac{1}{g(+\infty)}, \frac{1}{g\left(0^{+}\right)}\right)$. Since $r \longrightarrow \frac{1}{g(1 / r)}$ is a bijection from $(0,+\infty)$ onto $\left(r_{0}, r_{1}\right)$, there exists a (unique) $r>0$ such that $\rho=\frac{1}{g(1 / r)}$, i.e. $r=\frac{1}{g^{-1}(1 / \rho)}$, and

$$
\left\|T_{t}^{g} f\right\|_{2}^{2} \leq e^{-2 t / \rho}\|f\|_{2}^{2}+\beta\left(\frac{1}{g^{-1}(1 / \rho)}\right)\|f\|_{1}^{2}\left(1-e^{-2 t / \rho}\right) .
$$


We conclude by applying (ii) $\Rightarrow$ (i) of Proposition 2.1 with $g(A)$. Theorem 1.1 is proved.

Note that we do not need the existence of the density of the measures $\nu_{t}^{g}$ nor additional properties of the function $\beta$.

\subsection{Proof of Theorem 1.2}

Proof of (i). We assume that $A$ satisfies Nash-type inequality. By (ii) of Proposition 2.2 and the definition of $\beta$, we get super-Poincaré inequality: for any $f \in \mathcal{D}(A) \cap L^{1}(\mu)$,

$$
\|f\|_{2}^{2} \leq r(A f, f)+\beta(r)\|f\|_{1}^{2}, r>0,
$$

We now apply Theorem 1.1 and deduce super-Poincaré inequality for $g(A)$, i.e.

$$
\|f\|_{2}^{2} \leq r(g(A) f, f)+\beta_{g}(r)\|f\|_{1}^{2},
$$

with $\beta_{g}(r)=\beta\left(\frac{1}{g^{-1}(1 / r)}\right), r \in\left(r_{0}, r_{1}\right)=\left(\frac{1}{g(+\infty)}, \frac{1}{g\left(0^{+}\right)}\right)$. Now, we conclude by applying (i) of Proposition 2.2.

Proof of (ii). From the next lemma which compares $D_{g, \beta}$ and $g \circ D$ and (i), we immediately deduce the inequality (9).

Lemme 2.1 Let $g:(0,+\infty) \rightarrow(0,+\infty)$ be a bijective continuous increasing concave function (e.g. bijective Bernstein function), $D$ and $D_{g, \beta}$ defined as in Theorem 1.2 with $\beta$ a decreasing differentiable bijection from $(0,+\infty)$ to itself. Then for any $x>0$ and $\rho>1$,

$$
\left(1-\rho^{-1}\right)(g \circ D)\left(\rho^{-1} x\right) \leq D_{g, \beta}(x) \leq g \circ D(x) .
$$

Proof: To simplify our discussion, we set $V(t):=\beta(1 / t), V_{g}(t):=\beta_{g}(1 / t)=\beta\left(\frac{1}{g^{-1}(t)}\right)$ for $t>0$. So, $D(x)=\sup _{t>0}\left(t-\frac{t}{x} V(t)\right)$ and $D_{g, \beta}(x)=\sup _{t>0}\left(t-\frac{t}{x} V_{g}(t)\right)$ for $x>0$. As a consequence of the assumptions $\beta\left(0^{+}\right)=+\infty$, we have that $D$ and $D_{g, \beta}$ are well defined and finite on $(0,+\infty)$.

Let $u>0$. Since $g$ is a bijection from $(0,+\infty)$ to itself, there exists a unique $t>0$ such that $\frac{1}{u}=\frac{1}{g^{-1}(t)}$, i.e $t=g(u)$. Thus $D_{g, \beta}$ can be written as

$$
D_{g, \beta}(x)=\sup _{u>0} g(u)\left(1-\frac{V(u)}{x}\right) .
$$

Since $D=D_{i d}$ and by continuity of $g$, we get

$$
g \circ D(x)=\sup _{\{u>0: V(u) \leq x\}} g\left(u\left[1-\frac{V(u)}{x}\right]\right) .
$$

Let $a=1-V(u) / x$. Since $V \geqslant 0$, it is sufficient to consider $a \in(0,1)$. By concavity of $g$ and $g(0)=0$, we have $a g(u)=a g(u)+(1-a) g(0) \leq g(a u)$. Therefore, we conclude $D_{g, \beta}(x) \leq g \circ D(x)$ for any $x>0$.

Now, we prove the lower bound on $D_{g, \beta}$. From the definition, we have for any $x, u>0$,

$$
D_{g, \beta}(x) \geqslant g(u)\left(1-\frac{V(u)}{x}\right) .
$$


By the assumptions on $\beta$, the function $V$ is a differentiable increasing bijection from $(0,+\infty)$ to itself. Fix $x>0$. For $\rho>1$, we set $u=V^{-1}\left(\rho^{-1} x\right)$. It yields

$$
D_{g, \beta}(x) \geqslant\left(1-\rho^{-1}\right) g\left(V^{-1}\left(\rho^{-1} x\right)\right) .
$$

Fix $y>0$. The supremum defining $D(y)=t_{0}-t_{0} y^{-1} V\left(t_{0}\right)$ exists and it is attained at some point $t_{0}>0$ which is characterized by $1-\frac{1}{y} V\left(t_{0}\right)-\frac{t_{0}}{y} V^{\prime}\left(t_{0}\right)=0$. It implies that $y=V\left(t_{0}\right)+t_{0} V^{\prime}\left(t_{0}\right) \geqslant V\left(t_{0}\right)$ because $V^{\prime} \geqslant 0$. Finally, we get $t_{0} \leq V^{-1}(y)$. Since $V \geqslant 0$, we deduce $D(y) \leq t_{0} \leq V^{-1}(y)$. Thus $g(D(y)) \leq g\left(V^{-1}(y)\right)$ for any $y>0$. Now set $y=\rho^{-1} x$ and obtain the expected lower bound

$$
D_{g, \beta}(x) \geqslant\left(1-\rho^{-1}\right)(g \circ D)\left(\rho^{-1} x\right) .
$$

The proof is complete.

\section{Application to ultracontractivity of subordinated semigroups}

Recall that a symmetric semigroup $\left(T_{t}\right)_{t>0}$ of contraction on $L^{2}(\mu)$ and $L^{1}(\mu)$ is ultracontractive if for any $t>0$,

$$
\left\|T_{t} f\right\|_{2} \leq b(t)\|f\|_{1}
$$

for some non-increasing function $b:(0,+\infty) \rightarrow(0,+\infty)$ with $b\left(0^{+}\right)=+\infty$, see [D]. Ultracontractivity implies super-Poincaré (1) with $\beta(r)=b^{2}(r / 2)$. Indeed, since $s \rightarrow$ $\left(A T_{s} f, T_{s} f\right)$ is non-increasing, we get for any $r>0$,

$$
\|f\|_{2}^{2}-b^{2}(r / 2)\|f\|_{1}^{2} \leq\|f\|_{2}^{2}-\left\|T_{r / 2} f\right\|_{2}^{2}=\left(f-T_{r} f, f\right)=\int_{0}^{r}\left(A T_{s} f, T_{s} f\right) d s \leq r(A f, f) .
$$

Which is the desired inequality.

By interpolation and duality, the property of ultracontractivity is equivalent to

$$
\left\|T_{t} f\right\|_{\infty} \leq a(t)\|f\|_{1}, \quad t>0
$$

for some non-increasing function $a:(0,+\infty) \rightarrow(0,+\infty)$ with $a\left(0^{+}\right)=+\infty$. More precisely, from (14) we get $a(t) \leq b^{2}(t / 2)$ and from (15) we obtain $b(t) \leq \sqrt{a(t)}$.

If $b_{g}(t):=\int_{0}^{+\infty} b(s) \eta_{t}^{g}(s) d s<+\infty$ for any $t>0$ then the semigroup $\left(T_{t}^{g}\right)$ is ultracontractive since

$$
\left\|e^{-t g(A)} f\right\|_{2} \leq \int_{0}^{+\infty} \eta_{t}^{g}(s)\left\|T_{s} f\right\|_{2} d s \leq\left(\int_{0}^{+\infty} b(s) \eta_{t}^{g}(s) d s\right)\|f\|_{1} .
$$

But unfortunately, to check this condition is rather hard because the densities $\eta_{t}^{g}$ are not well known apart from the case of $g(x)=\sqrt{x}$, see [J] p.181. A way to overcome this difficulty is by considering Nash-type inequalities. For that purpose, we recall a result due to T.Coulhon. The author deduces ultracontractivity bounds from Nash-type inequality under some integrability condition, see [C] and also [M1]. For applications we have in mind, we restrict his result to our setting. 
Theorem 3.1 Let $\left(T_{t}\right)_{t>0}$ be a semigroup as in Section 1 with infinitesimal generator $-A$. Assume that there exists a non-decreasing function $\Theta:(0,+\infty) \rightarrow(0,+\infty)$ satisfying the following Nash-type inequality

$$
\Theta\left(\|f\|_{2}^{2}\right) \leq(A f, f), \quad\|f\|_{1} \leq 1 .
$$

If $\int^{\infty} \frac{d x}{\Theta(x)}<+\infty$ then $\left(T_{t}\right)_{t>0}$ is ultracontractive and for any $t>0$,

$$
\left\|T_{t} f\right\|_{\infty} \leq a(t)\|f\|_{1}, \quad t>0
$$

where $a(t)$ is the inverse of the function $s \rightarrow \int_{s}^{\infty} \frac{d x}{\Theta(x)}$.

We apply this result to the eventual ultracontractivity of the subordinated semigroup $\left(T_{t}^{g}\right)$ and give a sufficient condition on $D_{g, \beta}$ of (8) to get ultracontractivity from a Nash-type inequality satisfied by $A$.

Corollary 3.2 Under the assumptions and notations of Theorem 1.2, let's assume that $\int^{\infty} \frac{d x}{x D_{g, \beta}(x)}<+\infty$. Then $\left(T_{t}^{g}\right)$ is ultracontractive and for any $t>0$,

$$
\left\|T_{t}^{g} f\right\|_{\infty} \leq a_{g}(t)\|f\|_{1}
$$

where $a_{g}$ is the inverse function of $s \rightarrow \int_{s}^{\infty} \frac{d x}{x D_{g, \beta}(x)}$.

Proof: Apply Theorems 1.2 and 3.1.

It is clear that $\left(T_{t}\right)_{t>0}$ can be ultracontractive but not $\left(T_{t}^{g}\right)$ for some Bernstein functions $g$. For instance, let $A=\Delta$ be the usual Laplacian on $\mathbb{R}^{n}$. Then $A$ satisfies a Nash-type inequality with rate function $D(x)=c x^{2 / n}$. Let $g(r)=\log (1+r)$. Then $\int^{\infty} \frac{d x}{x D_{g, \beta}(x)} \geqslant \int^{\infty} \frac{d x}{x \ln \left(1+c x^{2 / n}\right)}=+\infty$. This is obtained from the inequality $g \circ D(x) \geqslant$ $D_{g, \beta}(x)$ of Theorem 1.2 (ii). Now by a direct computation, we can show that $\left(T_{t}^{g}\right)$ is not ultracontractive for small $t>0$, see (18) below for details.

Applications to heat kernel bounds: Ultracontractivity insures the existence and uniform bounds of the heat kernel under some assumptions on $X$. For instance, if $X$ is a locally compact separable metric space with a Radon measure $\mu$ with full support then ultracontractivity of $\left(T_{t}^{g}\right)$ implies existence of the heat kernel $k_{t}^{g}$ with respect to the measure $\mu$, i.e.

$$
T_{t}^{g} f(x)=\int_{X} f(y) k_{t}^{g}(x, y) d \mu(y) .
$$

Furthermore, the kernel satisfies the uniform bound,

$$
\operatorname{essup}_{x, y \in X} k_{t}^{g}(x, y) \leq a_{g}(t), t>0 .
$$

See the recent paper [G-H] (Lemma 3.7) for a detailed exposition on the existence of the heat kernel. See also [D] Chap.2.

\section{Examples of Settings}

Here, we give some examples where our results can be applied. 


\subsection{The Euclidean space}

Let $\Delta=-\sum_{i=1}^{n} \frac{\partial^{2}}{\partial x_{i}^{2}}$ be the usual Laplacian on $\mathbb{R}^{n}$. The profile of the super-Poincaré inequality can be deduced from the optimal Nash inequality obtained in [C-L]. Let $N_{n}$ be the best constant in Nash inequality,

$$
\frac{1}{N_{n}}\|f\|_{2}^{2+4 / n} \leq(\Delta f, f), \quad\|f\|_{1} \leq 1 .
$$

By Proposition 2.2, this is equivalent to the following super-Poincaré inequality,

$$
\|f\|_{2}^{2} \leq r(\Delta f, f)+C_{n} r^{-n / 2}\|f\|_{1}^{2}, r>0,
$$

with

$$
C_{n}=\frac{2\left(n N_{n}\right)^{n / 2}}{(n+2)^{1+n / 2}}
$$

Thus (1) is satisfied with the super-Poincaré profile $\beta_{p}(r)=C_{n} r^{-n / 2}$ and (3) with $D(x)=$ $\frac{1}{N_{n}} x^{2 / n}$.

To simplify the presentation of our results, we shall assume that the Bernstein function $g$ is a bijection from $(0,+\infty)$ to itself. By applying (ii) of Theorem 1.2, we get for any $\rho>1$,

$$
\frac{1}{2}\left(1-\rho^{-1}\right)\|f\|_{2}^{2} g\left(2 N_{n}^{-1} \rho^{-2 / n}\|f\|_{2}^{4 / n}\right) \leq(g(\Delta) f, f), \quad\|f\|_{1} \leq 1 .
$$

Examples of Bernstein functions.

i. Let $g(x)=x^{\alpha}$. We obtain for the fractional power of the Laplacian $\Delta^{\alpha}, 0<\alpha<1$,

$$
\frac{1}{2\left(N_{n} \rho\right)^{\alpha}}\left(1-\rho^{-1}\right)\|f\|_{2}^{2+4 \alpha / n} \leq\left(\Delta^{\alpha} f, f\right), \quad\|f\|_{1} \leq 1 .
$$

By optimizing over $\rho>1$, we deduce

$$
L_{n, \alpha}\|f\|_{2}^{2+4 \alpha / n} \leq\left(\Delta^{\alpha} f, f\right), \quad\|f\|_{1} \leq 1,
$$

with $L_{n, \alpha}=2^{\alpha-1} N_{n}{ }^{-\alpha} \frac{n(2 \alpha)^{2 \alpha / n}}{(2 \alpha+n)^{1+2 \alpha / n}}$.

See [VSC] for such a result in the setting of sub-markovian symmetric semigroups but with a different approach. Note that the constant $L_{n, \alpha}$ is explicit but probably not optimal. Indeed, we get a better constant if we apply Theorem 1.1 with $g(x)=x^{\alpha}$ :

$$
\|f\|_{2}^{2} \leq r\left(\Delta^{\alpha} f, f\right)+C_{n} 2^{\frac{n}{2}\left(\frac{1}{\alpha}-1\right)} r^{-\frac{n}{2 \alpha}}\|f\|_{1}^{2}, r>0,
$$

with $C_{n}$ as above. By applying (i) of Proposition 2.2, we get

$$
K_{n, \alpha}\|f\|_{2}^{2+4 \alpha / n} \leq\left(\Delta^{\alpha} f, f\right), \quad\|f\|_{1} \leq 1 .
$$

with

$$
K_{n, \alpha}=\left(\frac{n}{n+2 \alpha}\right) 2^{\alpha-1}\left(\left(\frac{n}{2 \alpha}+1\right) C_{n}\right)^{\frac{-2 \alpha}{n}} .
$$

By the relationships connecting $N_{n}$ and $C_{n}$, we have that $L_{n, \alpha}<K_{n, \alpha}$ (equivalent to the trivial inequality $\left.n 2^{\frac{2}{n}}<(n+2)^{\frac{2}{n}+1}\right)$. We postpone to Section 7 the study of super-Poincaré inequalities for a larger class of functions of the Laplacian using Fourier analysis tools. But with this approach, the best constants are lost. 
ii. Let $g(x)=\log (1+x)$. The geometrically stable operator $\log (I+\Delta)$ satisfies

$$
\frac{1}{2}\left(1-\rho^{-1}\right)\|f\|_{2}^{2} \log \left(1+N_{n}^{-1} \rho^{-1}\|f\|_{2}^{4 / n}\right) \leq(\log (I+\Delta) f, f), \quad\|f\|_{1} \leq 1 .
$$

To estimate $D_{g, \beta}$ with $g(x)=\log (1+x)$ is not a pleasant task. So, we prefer to state this explicit inequality for each parameter $\rho>1$.

Note that, in general, the eventual ultracontractivity can be proved for $e^{-t g(\Delta)}$ directly by the formula,

$$
\left\|e^{-t g(\Delta)}\right\|_{1 \rightarrow 2}^{2}=\frac{1}{(2 \pi)^{n}} \int_{\mathbb{R}^{n}} e^{-2 t g\left(|y|^{2}\right)} d y .
$$

Applied to $g(x)=\log (1+x)$, this leads us to

$$
\left\|e^{-t \log (I+\Delta)}\right\|_{1 \rightarrow 2}^{2}=\frac{\left|S_{n-1}\right|}{(2 \pi)^{n}} \int_{0}^{\infty}\left(1+r^{2}\right)^{-2 t} r^{n-1} d r<+\infty \quad \text { iff } \quad t>\frac{n}{4} .
$$

Thus this semigroup is not ultracontractive for $0<t \leq n / 4$. But note that it satisfies super-Poincaré and Nash-type inequalities.

\subsection{The Riemannian setting}

The following example is taken from [W2] Cor.2.5. Let $M$ be a connected complete Riemannian manifold with Ricci curvature bounded from below. Assume that the boundary $\partial M$ is convex or empty. For $V \in C^{1}(M)$, we assume $Z=\int_{M} e^{-V(x)} d x$ is finite and define the probability measure $\mu$ by $d \mu(x)=Z^{-1} e^{-V(x)} d x$ where $d x$ is the Riemannian volume measure. Let $A=\Delta+\nabla V, A$ is (essentially) self-adjoint on $L^{2}(\mu)$ (with Neumann boundary condition whenever $\partial M$ is nonempty). Set $\rho(x)=\rho(x, o)$ the Riemannian distance function to a fixed point $o \in M$. Consider $V=-\alpha \rho^{\delta}, \alpha>0$ and $\delta>1$ then super-Poincaré (1) holds true with

$$
\beta(r)=\exp \left[c\left(1+r^{-\lambda}\right)\right]
$$

with $\lambda=\delta /[2(\delta-1)]$ and some constant $c>0$. Moreover, a super-Poincaré holds if $V=-\exp [\alpha \rho], \alpha>0$ with $\lambda=1 / 2$ and the rate function $\beta$ given in (19). Theorem 1.1 implies that $g(A)$ satisfies a super-Poincaré with rate function $\beta_{g}(r)$ given in Theorem 1.1. Nash-type inequalities can be deduced for $g(A)$ from super-Poincaré by Theorem 1.2. Our result generalize the particular case of the fractional powers $g(x)=x^{\alpha}, 0<\alpha<1$ treated in $[\mathrm{W} 1]$.

\subsection{The hypoelliptic setting}

Here, we consider sub-laplacians on Lie groups of polynomial growth. Let $G$ be a connected Lie group of polynomial growth of index $D$ and $\left(X_{1}, X_{2}, \ldots, X_{m}\right)$ be a system of leftinvariant vector fields satisfying Hörmander's condition with local dimension $d$. We assume $d \leq D$. The sub-laplacian $L=-\sum_{i=1}^{m} X_{i}^{2}$ generates a semigroup $e^{-t L}$ with density kernel $p_{t}$ satisfying for all $n$ satsifying $d \leq n \leq D$,

$$
\sup _{x, y \in G} p_{t}(x, y)=\left\|e^{-t L}\right\|_{1 \rightarrow \infty} \leq \frac{c_{1}}{t^{n / 2}} .
$$


Hence, a super-Poincaré inequality holds true

$$
\|f\|_{2}^{2} \leq t(L f, f)+\frac{c_{0}}{t^{n / 2}}\|f\|_{1}^{2}, \quad t>0 .
$$

and our results applies to $g(L)$ for any Bernstein function $g$ with $\beta(t)=c_{0} t^{-n / 2}$, see [VSC]. We now discuss in this context the four examples of Bernstein function $g$ introduced below Theorem 1.1. We provide asymptotic behaviors of $\beta_{g}(r)$ when $r$ tends to 0 and $r$ tends to $+\infty$.

i. If $g(x)=x^{\alpha}, 0<\alpha \leq 1$ then $\beta_{g}(r)=\frac{c_{0}}{r^{n / 2 \alpha}}, r>0$.

ii. If $g(x)=\ln (1+x)$ then $\beta_{g}(r)=c_{0}\left(e^{1 / r}-1\right)^{n / 2}, r>0$.

$$
\beta_{g}(r) \sim\left\{\begin{array}{l}
c_{0} e^{n / 2 r} \text { as } r \rightarrow 0^{+} \\
c_{0} \frac{1}{r^{n / 2}} \text { as } r \rightarrow+\infty
\end{array}\right.
$$

iii. If $g(x)=\left[\ln \left(1+x^{\alpha}\right)\right]^{\gamma}, 0<\alpha, \gamma \leq 1$ then $\beta_{g}(r)=c_{0}\left[e^{(1 / r)^{1 / \gamma}}-1\right]^{n / 2 \alpha}, r>0$.

$$
\beta_{g}(r) \sim \begin{cases}c_{0} e^{\frac{n}{2 \alpha}(1 / r)^{1 / \gamma}} & \text { as } r \rightarrow 0^{+}, \\ c_{0} \frac{1}{r^{n / 2 \alpha \gamma}} & \text { as } r \rightarrow+\infty .\end{cases}
$$

iv. Let $t>0$. If $g(x)=1-e^{-t x}$ then $\beta_{g}(r)=\frac{c_{0}}{t^{n / 2}}\left[\ln \left(1+\frac{1}{r-1}\right)\right]^{n / 2}, r>1$.

$$
\beta_{g}(r) \sim\left\{\begin{array}{l}
\frac{c_{0}}{t^{n / 2}}\left[\ln \left(\frac{1}{r-1}\right)\right]^{n / 2} \text { as } r \rightarrow 1^{+}, \\
\frac{c_{0}}{(r t)^{n / 2}} \text { as } r \rightarrow+\infty
\end{array}\right.
$$

Note that this discussion with this family of Bernstein functions is always valid when $A$ satisfies super-Poincaré inequality with $\beta(t)=c_{0} t^{-n / 2}$.

\subsection{The Ornstein-Uhlenbeck operator}

Let $A=\mathcal{L}=\Delta+x . \nabla$ be the Ornstein-Uhlenbeck operator define on $L^{2}\left(\mathbb{R}^{n}, \gamma\right)$ with the gaussian measure $\gamma(d x)=(2 \pi)^{-n / 2} e^{-\frac{|x|^{2}}{2}} d x$. It is well known that Gross' logarithmic Sobolev inequality is satisfied

$$
\int_{\mathbb{R}^{n}} f^{2} \log \left(|f| /|| f \|_{2}\right) d \gamma \leq(\mathcal{L} f, f)
$$

with $(\mathcal{L} f, f)=\int_{\mathbb{R}^{n}}|\nabla f|^{2} d \gamma$.

A super-Poincaré inequality can be deduced from Logarithmic Sobolev inequality. We recall the arguments. For any $f \in L^{1}(\mu) \cap L^{2}(\mu)$ such that $\|f\|_{1}=1$, we have by Jensen's inequality:

$$
\|f\|_{2}^{2} \log \|f\|_{2} \leq \int_{\mathbb{R}^{n}} f^{2} \log \left(|f| /|| f \|_{2}\right) d \gamma .
$$

By renormalization, this inequality is also satisfied when $\|f\|_{1} \leq 1$. This yields

$$
\|f\|_{2}^{2} \log \|f\|_{2} \leq(\mathcal{L} f, f) .
$$


Using the relation $x y-e^{y-1} \leq x \log x$ for any $y \in \mathbb{R}$, we deduce

$$
\|f\|_{2}^{2} \leq t(\mathcal{L} f, f)+\frac{t}{2 e} e^{\frac{2}{t}}\|f\|_{1}^{2}, t>0
$$

On the other hand, Poincaré inequality deduced from Gross' inequality, trivially implies $\|f\|_{2}^{2} \leq(\mathcal{L} f, f)+\|f\|_{1}^{2}$. Together, the preceding inequalities leads to the following formulation of super-Poincaré inequality

$$
\|f\|_{2}^{2} \leq t(\mathcal{L} f, f)+\beta(t)\|f\|_{1}^{2}, t>0
$$

with $\beta(t)=\frac{t}{2 e} e^{\frac{2}{t}}, 0<t \leq 1$ and $\beta(t)=1, t \geqslant 1$.

For instance Theorem 1.1 implies :

i. For any $0<\alpha<1$,

$$
\|f\|_{2}^{2} \leq t\left(\mathcal{L}^{\alpha} f, f\right)+\frac{t^{\frac{1}{\alpha}}}{2 e} e^{2 t^{-1 / \alpha}}\|f\|_{1}^{2}, \quad 0<t<1
$$

and

$$
\|f\|_{2}^{2} \leq t\left(\mathcal{L}^{\alpha} f, f\right)+\|f\|_{1}^{2}, \quad t \geqslant 1
$$

ii.

$$
\|f\|_{2}^{2} \leq t(\log (I+\mathcal{L}) f, f)+\frac{1}{2 e^{3}} \frac{1}{\left(e^{1 / t}-1\right)} e^{2 e^{1 / t}}\|f\|_{1}^{2}, \quad 0<t<\frac{1}{\log 2},
$$

and

$$
\|f\|_{2}^{2} \leq t(\log (I+\mathcal{L}) f, f)+\|f\|_{1}^{2}, \quad t \geqslant \frac{1}{\log 2} .
$$

we have $\beta_{\log }(t) \sim \frac{1}{2 e^{3}} e^{2 e^{1 / t}-1 / t}$ as $t$ goes to 0.

Similar inequalities can be written for the cases (iii) and (iv) considered in Section 1. Of course, the discussion is not limited to these cases just above.

\section{$5 \quad$ Study of $\Psi(A)$ with $\Psi$ convex}

It is useful to deduce super-Poincaré or Nash inequality for $\Psi(A)$ when $A$ satisfies such inequality and $\Psi$ is convex. The reason is that the inverse function of a concave increasing function is convex and increasing. For instance, Bernstein functions. In what follows, we study the following converse implication. Assume that $g(A)$ satisfies super-Poincaré or Nash-type inequality then deduce a similar inequality for $A$.

Let $\left(E_{\lambda}\right)_{\lambda>0}$ be the spectral resolution associated to $A$ and $\psi:[0, \infty) \rightarrow[0, \infty)$ be a measurable function. We define $\psi(A)$ on its domain $\mathcal{D}(\psi(A)) \subset L^{2}(\mu)$ as in Section 1. In particular, we have on their respective domains the following representations

$$
(A f, f)=\int_{0}^{+\infty} \lambda d\left(E_{\lambda} f, f\right), \quad\left(T_{t} f, f\right)=\int_{0}^{+\infty} e^{-\lambda t} d\left(E_{\lambda} f, f\right), \quad\|f\|_{2}^{2}=\int_{0}^{+\infty} d\left(E_{\lambda} f, f\right) .
$$

See $[\mathrm{Sc}-\mathrm{S}-\mathrm{V}]$ Thm. 11.4. 
Proposition 5.1 Assume that $A$ is a non-negative self-adjoint operator satisfying Nashtype inequality (3). Then for any non-negative non-decreasing convex function $\Psi$ with $\Psi(0)=0$, we have

$$
\|f\|_{2}^{2}(\Psi \circ D)\left(\|f\|_{2}^{2}\right) \leq(\Psi(A) f, f), \quad\|f\|_{1} \leq 1 .
$$

Note that such result can be generalized in the framework of Hilbert space $H$ with the norm $\|f\|_{1}$ replaced by another control $\Phi(f), f$ in some subspace of $H$, satisfying properties as defined in [W4].

Proof: Write equivalently Nash-type inequality (3) as follows. For any $f \in \mathcal{D}(A) \cap$ $L^{1}(\mu)$ and $\|f\|_{2}=1$,

$$
D\left(\frac{1}{\|f\|_{1}^{2}}\right) \leq(A f, f) .
$$

Since $\Psi$ is non-decreasing, we get

$$
(\Psi \circ D)\left(\frac{1}{\|f\|_{1}^{2}}\right) \leq \Psi[(A f, f)] .
$$

Now, by functional calculus and Jensen's inequality applied to the probability measure $d\left(E_{\lambda} f, f\right)$, i.e. $\|f\|_{2}=1$, we get

$$
\Psi[(A f, f)]=\Psi\left(\int_{0}^{+\infty} \lambda d\left(E_{\lambda} f, f\right)\right) \leq \int_{0}^{+\infty} \Psi(\lambda) d\left(E_{\lambda} f, f\right)=(\Psi(A) f, f) .
$$

Thus

$$
(\Psi \circ D)\left(\frac{1}{\|f\|_{1}^{2}}\right) \leq(\Psi(A) f, f), \quad\|f\|_{2}=1, f \in \mathcal{D}(A) \cap L^{1}(\mu) .
$$

Under the assumptions on $\Psi$, there exists $\lambda_{0}>0$ and $k>0$ such that $k \lambda \leq \Psi(\lambda)$ for any $\lambda>\lambda_{0}$. This implies $\mathcal{D}(\Psi(A)) \subset \mathcal{D}(A)$. Now, by reversing the process of normalization from $L^{2}(\mu)$ to $L^{1}(\mu)$, it yields for any $f \in \mathcal{D}(\Psi(A)) \cap L^{1}(\mu)$,

$$
\|f\|_{2}^{2}(\Psi \circ D)\left(\frac{\|f\|_{2}^{2}}{\|f\|_{1}^{2}}\right) \leq(\Psi(A) f, f) .
$$

Since $\Psi \circ D$ is non-increasing, we deduce (21) for $\|f\|_{1} \leq 1$ and conclude the proof.

In the second part of this section, we deal with the case of super-Poincaré inequalities generalizing the arguments of [W1] used for $A^{\alpha}, \alpha>1$.

Theorem 5.2 Let $\Psi:(0,+\infty) \rightarrow(0,+\infty)$ be a non-decreasing convex function. Assume that $\Psi^{*}(x):=\sup _{y \in(0,+\infty)}(x y-\Psi(y))$ is a bijection from $(0,+\infty)$ to $(0,+\infty)$ and that $B$ is a non-negative symmetric operator satisfying a super-Poincaré inequality with some rate function $\gamma$.

Then $\Psi(B)$ satisfies also a super-Poincaré inequality with rate function, for $t>0$,

$$
\gamma_{\Psi}(t)=\inf _{0<\varepsilon<1} \frac{1}{\varepsilon} \gamma\left(\varepsilon t\left(\Psi^{*}\right)^{-1}\left(\frac{1-\varepsilon}{\varepsilon t}\right)\right) .
$$

In particular, if $\Psi(x)=x^{1 / \alpha}$ with $\alpha \in(0,1)$ then $\gamma_{\Psi}(t) \leq \frac{1}{\alpha} \gamma\left(t^{\alpha}\right)$. 
Proof: Young's inequality, for any $y, s>0, y s \leq \Psi(y)+\Psi^{*}(s)$ implies for $y=(B f, f)$ with $f \in \mathcal{D}(B)$, and $s>0$,

$$
s(B f, f) \leq \Psi((B f, f))+\Psi^{*}(s) .
$$

Assume that $\|f\|_{2}=1$. By spectral representation of $B$ and by Jensen's inequality with $\Psi$ as convex function, we have already seen that

$$
0 \leq \Psi((B f, f)) \leq(\Psi(B) f, f) .
$$

Super-Poincaré implies that for any $t, s>0$ with $\|f\|_{2}=1$,

$$
1 \leq t s(B f, f)+\gamma(t s)\|f\|_{1}^{2} .
$$

Combining the above inequalities, it yields for $f \in \mathcal{D}(\Psi(B))$,

$$
1 \leq t(\Psi(B) f, f)+t \Psi^{*}(s)+\gamma(t s)\|f\|_{1}^{2} .
$$

Let $\varepsilon \in(0,1)$. Since $\Psi^{*}$ is a bijection, for any fixed $t>0$, there exists $s>0$ such that $\varepsilon=1-t \Psi^{*}(s)$, i.e. $s=\left(\Psi^{*}\right)^{-1}\left(\frac{1-\varepsilon}{t}\right)$. Thus we obtain

$$
\varepsilon \leq t(\Psi(B) f, f)+\gamma\left(t\left(\Psi^{*}\right)^{-1}\left((1-\varepsilon) t^{-1}\right)\right)\|f\|_{1}^{2} .
$$

Changing $t$ by $\varepsilon t$ and dividing by $\varepsilon$, we get for any $t>0, \varepsilon \in(0,1)$ and $\|f\|_{2}=1$,

$$
1 \leq t(\Psi(B) f, f)+\frac{1}{\varepsilon} \gamma\left(\varepsilon t\left(\Psi^{*}\right)^{-1}\left(\frac{1-\varepsilon}{\varepsilon t}\right)\right)\|f\|_{1}^{2} .
$$

We conclude by changing $f$ by $f /\|f\|_{2}$ and by taking the infimum over $\varepsilon \in(0,1)$.

We now prove the last statement. Let $\Psi(x)=x^{1 / \alpha}$, we have $\Psi^{*}(s)=c_{\alpha} s^{\frac{1}{1-\alpha}}$ with $c_{\alpha}=(1-\alpha) \alpha^{\frac{\alpha}{1-\alpha}}$. A simple computation yields for any $\varepsilon \in(0,1)$,

$$
\gamma_{\Psi}(t) \leq \frac{1}{\varepsilon} \gamma\left[k_{\alpha} \varepsilon\left(\frac{1-\varepsilon}{\varepsilon}\right)^{1-\alpha} t^{\alpha}\right]
$$

with $k_{\alpha}=\alpha^{-\alpha}(1-\alpha)^{\alpha-1}$. Choosing $\varepsilon=\alpha$, we conclude $\gamma_{\Psi}(t) \leq \frac{1}{\alpha} \gamma\left(t^{\alpha}\right)$. The proof is complete.

For the case $g(x)=x^{\alpha}$, the function obtained in [W1] is given by $\tilde{\gamma}_{\Psi}(t)=2 \gamma\left(\frac{t^{\alpha}}{2}\right)$. Since $\gamma_{\Psi}$ is usually decreasing and $\gamma\left(0^{+}\right)=+\infty$, the result above is sharper up to a multiplicative constant. We notice that $\|f\|_{1}^{2}$ plays no particular role in the proof. So, it can be replaced by some functional $\Phi(f)$ and $L^{2}(\mu)$ by a general Hilbert space as in [W1].

Now we make the connection between Bernstein functions and convex functions. Assume that $g$ is a Bernstein function. Since $g$ is non-decreasing and concave, $\Psi=g^{-1}$ is non-decreasing and convex. Hence, Theorem 5.2 allows us to prove a converse to Theorem 1.1 about super-Poincaré inequalities. Thus applying Theorem 5.2 with $B=g(A)$, we get:

Corollary 5.3 Let $g$ be a bijective Bernstein function and $A$ be a non-negative symmetric operator. Assume that $\Psi=g^{-1}$ satisfies the conditions of Theorem 5.2 and that that $g(A)$ satisfies a super-Poincaré inequality with some rate function $\gamma$.

Then A satisfies a super-Poincaré inequality with rate function $\gamma_{\Psi}$ given in Theorem 5.2. 
Corollary 5.3 is sharp in the particular case $g(x)=x^{\alpha}, \alpha \in(0,1)$. Indeed, assume that $A$ satisfies super-Poincaré inequality with rate function $\beta$. By Theorem 1.1, $g(A)$ satisfies super-Poincaré inequality with $\gamma=\beta_{g}$ given in Theorem 1.1. Now take $\Psi(x)=x^{1 / \alpha}$ in Theorem 5.2, it gives back that $A$ satisfies super-Poincaré inequality with $\tilde{\beta}(t)=\gamma_{\Psi}(t) \leq$ $\frac{1}{\alpha} \gamma\left(t^{\alpha}\right)=\frac{1}{\alpha} \beta(t)$. In this case, Corollary 5.3 is essentially an optimal converse of Theorem 1.1 up to the multiplicative constant $\frac{1}{\alpha}$.

\section{Asymptotic behavior of $g(A)$}

We briefly revisit the relation between the asymptotic behavior of $g(A)$ and the asymptotic behavior of $A$ in terms of Poincaré inequality (equivalent to a bound on the bottom of the spectrum). The proof uses arguments of Theorem 1.1. We obtain Poincaré inequality in $L^{p}(\mu)$ for $g(A)$ starting from the same inequality for $A$. See $[\mathrm{C}-\mathrm{G}-\mathrm{R}]$ for recent results on the subject on $L^{p}$.

Proposition 6.1 Assume that $\left(T_{t}\right)_{t>0}$ is a symmetric Markov semigroup satisfying the following inequality

$$
\left\|T_{t} f-\mu(f)\right\|_{p} \leq e^{-\lambda t} C(f), \quad t>0,
$$

for some $\lambda \in[0,+\infty), p>1$ and some positively homogeneous functional $C(f)$. Then for any Bernstein function $g$ with $g(0)=0$, we have

$$
\left\|T_{t}^{g} f-\mu(f)\right\|_{p} \leq e^{-t g(\lambda)} C(f), \quad t>0 .
$$

For instance with $C(f)=\|f-\mu(f)\|_{p}$, see $[\mathrm{C}-\mathrm{G}-\mathrm{R}]$. For $p=2$ and $C(f)=\|f-\mu(f)\|_{2}$, (22) is the classical Poincaré inequality

$$
\|f-\mu(f)\|_{2}^{2} \leq \frac{1}{\lambda}(A f, f), \quad f \in \mathcal{D}(A) .
$$

Then similarly for $g(A)$, we deduce the Poincaré inequality for $g(A)$,

$$
\|f-\mu(f)\|_{2}^{2} \leq \frac{1}{g(\lambda)}(g(A) f, f) .
$$

Proof: Let $f$ such that $\mu(f)=0$. Then $\mu\left(T_{s} f\right)=\mu\left(T_{s}^{g} f\right)=0, s>0$ since $T_{s}$ and $T_{s}^{g}$ are symmetric. We have for any $t>0$,

$$
\begin{aligned}
& \left\|T_{t}^{g} f-\mu(f)\right\|_{p}=\left\|T_{t}^{g} f\right\|_{p}=\left\|\int_{0}^{+\infty} \eta_{t}^{g}(s) T_{s} f d s\right\|_{p} \\
& \leq \int_{0}^{+\infty} \eta_{t}^{g}(s)\left\|T_{s} f\right\|_{p} d s \leq C(f) \int_{0}^{+\infty} \eta_{t}^{g}(s) e^{-\lambda s} d s \leq e^{-t g(\lambda)} C(f) .
\end{aligned}
$$

This concludes the proof.

\section{$7 \quad$ Functions of the Laplacian on $\mathbb{R}^{n}$}

Here, we give a direct proof of super-Poincaré inequality for $g(\Delta)$ with $g$ a Bernstein function and $\Delta$ the usual Laplacian on $\mathbb{R}^{n}$. In fact, $g$ need not be a Bernstein function. The constants are certainly not optimal. The proof follows the original idea used for the 
Laplacian in the paper by J. Nash $[\mathrm{N}]$.

We use the following definition of Fourier transform $\mathcal{F} f(x)=\int_{\mathbb{R}^{n}} f(y) e^{-i x y} d y$. Let $\Delta=-\sum_{i=1}^{n} \frac{\partial^{2}}{\partial^{2} x_{i}}$. So, $\mathcal{F}(\Delta f)(x)=|x|^{2} \mathcal{F} f(x)$. The function $g(\Delta) f$ is defined by its Fourier transform

$$
\mathcal{F}(g(\Delta) f)(x)=g\left(|x|^{2}\right) \mathcal{F} f(x), x \in \mathbb{R}^{n} .
$$

The domain of $g(\Delta)$ is defined by

$$
\mathcal{D}(g(\Delta))=\left\{f \in L^{2}(\mu): \int_{\mathbb{R}^{n}}\left|g\left(|x|^{2}\right)\right|^{2}|\mathcal{F} f(x)|^{2} d x<+\infty\right\} .
$$

We denote by $\omega_{n}$ the volume of the unit ball of $\mathbb{R}^{n}$ and set $c_{n}=(2 \pi)^{-n}$.

Theorem 7.1 Let $g:[0,+\infty) \rightarrow[0,+\infty)$ be non-decreasing such that $g(0)=0$. Let define, for $u \in[0,+\infty), g \rightarrow(u)=\sup \{s \geqslant 0: g(s) \leq u\} \in[0,+\infty]$. Then we have

i. For any $t>0$ and any $f \in \mathcal{D}(g(\Delta)) \cap L^{1}\left(\mathbb{R}^{n}\right)$,

$$
\|f\|_{2}^{2} \leq t(g(\Delta) f, f)+\tilde{\beta}\left(\frac{1}{g \rightarrow\left(t^{-1}\right)}\right)\|f\|_{1}^{2}
$$

with $\tilde{\beta}(t)=c_{n} \omega_{n} t^{-n / 2}$.

ii. For any $f \in \mathcal{D}(g(\Delta)) \cap L^{1}\left(\mathbb{R}^{n}\right)$ with $\|f\|_{1} \leq 1$,

$$
\|f\|_{2}^{2} \tilde{D}_{g}\left(\|f\|_{2}^{2}\right) \leq(g(\Delta) f, f)
$$

with $\tilde{D}_{g}(x)=\sup _{t>0}\left(t-\frac{t}{x} c_{n} \omega_{n}[g \rightarrow(t)]^{n / 2}\right)$.

When $g$ is unbounded and $g(0)=0$, the function $g \rightarrow(t)$ is well defined and finite for any $t>0$. The generalized inverse function $g \rightarrow$ is non-decreasing and when $g$ is an increasing bijection we have $g^{\rightarrow}=g^{-1}$. The function $\tilde{\beta}_{g}(t):=\tilde{\beta}\left(\frac{1}{g^{\rightarrow\left(t^{-1}\right)}}\right)$ in (24) is similar to $\beta_{g}(t)$ in Theorem 1.1 when $g$ is invertible. If we assume that $g$ is bounded then $g(\Delta)$ is a bounded operator and $\tilde{\beta}_{g}(t)=+\infty$ when $t \leq 1 /\|g\|_{\infty}$. In that case, the inequality (24) is meaningful only for $t>1 /\|g\|_{\infty}$. Note that this restriction already appears in Theorem 1.1 when $g$ is bounded.

Proof: Let $f \in \mathcal{D}(g(\Delta)) \cap L^{1}(\mu)$ and $t>0$. By Plancherel formula,

$$
\begin{array}{r}
\|f\|_{2}^{2}=c_{n} \int_{\mathbb{R}^{n}}|\mathcal{F} f|^{2}(x) d x=c_{n} \int_{\left\{x \in \mathbb{R}^{n}: 1 \leq t g\left(|x|^{2}\right)\right\}}|\mathcal{F} f|^{2}(x) d x+c_{n} \int_{\left\{x \in \mathbb{R}^{n}: 1>\operatorname{tg}\left(|x|^{2}\right)\right\}}|\mathcal{F} f|^{2}(x) d x \\
\leq c_{n} t \int_{\mathbb{R}^{n}} g\left(|x|^{2}\right)|\mathcal{F} f|^{2}(x) d x+c_{n}\|\mathcal{F} f\|_{\infty}^{2} V\left(\left\{x \in \mathbb{R}^{n}: g\left(|x|^{2}\right)<\frac{1}{t}\right\}\right)
\end{array}
$$

where $V(\Omega)$ is the Lebesgue measure of the set $\Omega \subset \mathbb{R}^{n}$. Now, since $g(r) \leq u$ implies $r \leq g^{\rightarrow}(u)$ and $\|\mathcal{F} f\|_{\infty} \leq\|f\|_{1}$, we deduce for any $t>0$,

$$
\|f\|_{2}^{2} \leq t\left(g(\Delta f, f)+c_{n}\|f\|_{1}^{2} V\left(\left\{x \in \mathbb{R}^{n}:|x| \leq \sqrt{g^{\rightarrow}\left(t^{-1}\right)}\right\}\right) .\right.
$$

This concludes (i).

We prove the second part by applying Proposition 2.2 with $\tilde{\beta}(t)=c_{n} \omega_{n}\left[g^{\rightarrow}\left(t^{-1}\right)\right]^{n / 2}$. This completes the proof. 


\section{Appendix on Legendre transform}

In the first part of this section, we are interested to discuss the properties of $D$ directly from the properties of $\beta$ independently of the set $\mathcal{G}$ defined in Proposition 2.2. In the second part of this section, reversing the role of $\beta$ and $D$ leads to a similar discussion. The conditions introduced here are usually satisfied in the applications.

Lemma 8.1 Let $\beta$ be a non-negative function on $(0,+\infty)$ and set

$$
D(x)=\sup _{t>0}\left\{t-\frac{t}{x} \beta(1 / t)\right\} \in(-\infty,+\infty], \quad x>0 .
$$

i. If $\lim _{t \rightarrow 0^{+}} t \beta(1 / t)=0$ then $D$ is non-negative. This condition is satisfied if $\beta$ is bounded above at infinity, in particular if $\beta$ is non-increasing.

ii. If $\beta\left(0^{+}\right)=+\infty$ then $D(x)$ is finite for any $x>0$. Moreover, the function $x \rightarrow x D(x)$ is convex, non-decreasing on $(0,+\infty)$ and $D$ is continuous.

\section{Proof:}

i. Assume that $\lim _{t \rightarrow 0^{+}} t \beta(1 / t)=0$ then $D(x) \geqslant \lim _{t \rightarrow 0^{+}}\left(t-\frac{t}{x} \beta(1 / t)\right)=0$ for any $x>0$. Obviously, if $\beta$ is bounded above at infinity (e.g. non-increasing) and nonnegative then $\lim _{t \rightarrow 0^{+}} t \beta(1 / t)=0$.

ii. Assume $\beta\left(0^{+}\right)=+\infty$. Fix $x>0$ then there exists $t_{x}>0$ such that for any $t>t_{x}$, $x<\beta(1 / t)$. So, $t-\frac{t}{x} \beta(1 / t)<0$ when $t>t_{x}$. If $0<t \leq t_{x}$ then $t-\frac{t}{x} \beta(1 / t) \leq t_{x}$ since $\beta$ is non-negative. Therefore $D(x) \leq t_{x}$ and $D(x)$ is finite. Now, the function $x \rightarrow h^{*}(x)=x D(x)=\sup _{t>0}(t x-t \beta(1 / t))$ is convex on $(0,+\infty)$. Consequently, $h^{*}$ and $D$ are continuous.

The proof is complete.

Now, we study properties of $\beta$ in terms of $D$ defined as in Theorem 1.2. Natural conditions on $D$ comes from the previous lemma. The discussion is similar.

Lemma 8.2 Let $D:(0,+\infty) \rightarrow \mathbb{R}$ be a fixed function and set $\beta(r)=\sup _{x>0}\{x-r x D(x)\}$.

i. If $\lim _{x \rightarrow+\infty} D(x)=+\infty$ and $D$ is non-negative then $\beta(r)$ is finite for any $r>0$, convex, continuous and non-increasing.

ii. If $\lim _{x \rightarrow 0} x D(x)=0$ then $\beta$ is non-negative.

The proof is similar to Lemma 8.1. Indeed, let $\beta$ and $D$ as in Lemma 8.1. Note that $h^{*}(x):=x D(x)=\sup _{t>0}(t x-t \beta(1 / t))$ is the Lengendre transform (or complementary function) of $h(t)=t \beta(1 / t)$ thus the afferent theory applies. See for instance [R-R] p.6 for a discussion about Young functions and p.13 for the specific class of $\mathrm{N}$-functions. Usually $h$ is obtained from $h^{*}$ by the same formula, i.e. $h(t)=\sup _{x>0}\left(t x-h^{*}(x)\right), t>0$. In that case, we recover the definition of $\beta$ in terms of $D$ in Lemma 8.2 by the formulas $\beta(t)=t h(1 / t)$ and $D(x)=h^{*}(x) / x$. Here are some examples of couples of N-functions. They appear as asymptotics of functions $\beta$ or $D$ of our examples in Section 4 . Let $1<p, q<+\infty$ with $1 / p+1 / q=1$.

i. $\left(h_{1}(t), h_{1}^{*}(x)\right)=\left(t^{p} / p, x^{q} / q\right)$.

ii. $\left(h_{2}(t), h_{2}^{*}(x)\right)=\left(e^{t}-t-1,(1+x) \ln (1+x)-x\right)$.

iii. $\left(h_{3}(t), h_{3}^{*}(x)\right)=\left((1+t) \ln (1+t)-t, e^{x}-x-1\right)$. 
iv. $h_{4}(t)=e^{t^{p}}-1, h_{4}^{*}$ : no explicit form.

But one can prove that $h_{4}^{*}(x) \sim x(\ln x)^{1 / p}$ as $x \rightarrow+\infty$ and $h_{4}^{*}(x) \sim c_{q} x^{q}$ as $x \rightarrow 0^{+}$with $1 / p+1 / q=1$ and $c_{q}=(p-1)\left(\frac{1}{p}\right)^{q}$.

Of course, in applications, functions like $c_{1} h\left(c_{2} t\right), c_{i}>0$ should be considered or functions having asymptotics of this type. Indeed, in practice $h$ is not exactly an Nfunction but often close to such function. Fortunately, it doesn't cause much trouble in practice. This justifies the interest of both propositions just above. Now, we mentioned some cases where the asymptotics of these functions really appear in our applications. Recall the relations $\beta(t)=t h(1 / t)$ and $D(x)=h^{*}(x) / x$.

i. For $h_{1}(t)=c_{0} t^{p}$ then we get $\beta_{1}(t)=\frac{c_{1}}{t^{\nu}}$ where $\nu=p-1>0$ and $D(x)=c_{2} x^{q}$ with $1 / p+1 / q=1$ and $q=1+\frac{1}{\nu}$. Such cases correspond in Section 4.1 to the fractional Laplacian $\Delta^{\alpha}$ on the Euclidean space and to Section 4.3 with $L^{\alpha}$ where $L$ is a sum of vector fields satisfying Hörmander's condition and $\nu=\frac{n}{2 \alpha}$. For all these cases: $D(x)=c_{3} x^{\frac{2 \alpha}{n}}$.

ii. The function $h_{2}(t)=e^{t}-t-1$ leads to $\beta_{2}(t) \sim t e^{\frac{1}{t}}$ as $t \rightarrow 0^{+}$and $\beta_{2}(t) \sim \frac{1}{2 t}$ as $t \rightarrow+\infty$. This situation is realized up to multiplicative constants by the OrnsteinUhlenbeck operator in Section 4.4 as far as the local behavior, i.e. $t \rightarrow 0^{+}$, is concerned. In that case, $D(x) \sim \ln x$ as $x \rightarrow+\infty$.

iii. The function $h_{3}(t)=(1+t) \ln (1+t)-t$ gives $\beta_{3}(t) \sim \ln (1 / t)$ as $t \rightarrow 0^{+}$. Using results of Section 7 with $n=2$, we set $A=g(\Delta) \geqslant 0$ with $g(y)=e^{y / 4 \pi}-1, y \geqslant 0$. This provides an example of positive operator such that the super-Poincare inequality (24) is satisfied with $\beta(t)=\ln \left(1+\frac{1}{4 \pi^{2} t}\right), t>0$ and $D(x) \sim \frac{4 \pi^{2}}{x} e^{x-1}$ as $x \rightarrow+\infty$. We also have $D(x) \sim \pi^{2} x$ as $x \rightarrow 0^{+}$.

iv. For the function $h_{4}(t)=e^{t^{p}}-1$ with $1<p<+\infty$, we deduce $\beta_{4}(t) \sim t e^{\frac{1}{t^{p}}}=: \tilde{\beta}(t)$ as $t \rightarrow 0^{+}$and $e^{\left(1-\frac{1}{p}\right) \frac{1}{t^{p}}} \leq \tilde{\beta}(t) \leq e^{\frac{1}{t^{p}}}$ when $t \in(0,1)$. Examples with such behavior are given in the Riemannian setting of Section 4.2 with $p=\frac{\delta}{2(\delta-1)}$ where $1<\delta<2$ in (19). In that case, $D(x) \sim(\ln x)^{1 / p}$ as $x \rightarrow+\infty$. Note that it is a general fact that the behavior of $\beta(t)$ as $t \rightarrow 0^{+}$determines the behavior of $D(x)$ as $x \rightarrow+\infty$ and conversely.

It will be interesting to know if there exists an operator $A$ satisfying super-Poincaré inequality with $\beta(t) \sim t h_{3}(1 / t) \sim \ln (1 / t)$ as $t \rightarrow 0^{+}$and $(A f, f)$ a Dirichlet form.

Acknowlegments: The authors thank the anonymous referee for helpful comments and suggestions to improve the paper. This research was supported in part by the ANR project EVOL. The second author thanks the CNRS for a period of delegation during which this paper has been completed.

Note: The article [W1] can be obtained on demand to the author and difficult by other sources.

\section{References}

[B-M] Bendikov A.D. and Maheux P.: Nash type inequalities for fractional powers of nonnegative self-adjoint operators. Trans. Amer. Math. Soc. 359, no. 7 (2007), 3085-3097. 
[B-F] Berg Ch. and Forst G.: Potential theory on locally compact Abelian groups. Erg. der Math. und ihrer Grenzgeb., Band 87, Springer-Verlag 1975.

[Bi-M] Biroli M. and Maheux P.: Super Logarithmic Sobolev inequalities and Nash-type inequalities for sub-markovian symmetric semigroups. [hal-00465177, v1].

[C-L] Carlen E.A. and Loss M.: Sharp constant in Nash's inequality. Internat. Math. Res. Notices 1993, no. 7, 213-215.

[C-G-R] Cattiaux P., Guillin A. and Roberto C.: Poincaré inequality and the $L^{p}$ convergence of semi-groups. Electronic Communications in Probability, 2010, Vol.15, 270-280.

[C] Coulhon T.: Ultracontractivity and Nash type inequalities J.Funct.Anal.141 (1996), p.510-539.

[D] Davies E.B.: Heat kernels and spectral theory. Cambridge Tracts in Mathematics, 92. Cambridge University Press, Cambridge, 1989.

[G-H] Grigor'yan,A., Hu, J.: Upper bounds of heat kernels on doubling spaces. Preprint, 2010, available at http://www.math.uni-bielefeld.de/〜 grigor/pubs.htm.

[J] Jacob N.: Pseudo-Differential Operators and Markov Processes. Vol. 1: Fourier Analysis and Semigroups. Imperial College Press, London, 2001.

[M1] Maheux P.: New Proofs of Davies-Simon's Theorems about Ultracontractivity and Logarithmic Sobolev Inequalities related to Nash Type Inequalities. Preprint available on ArXiv: math/0609124.

[N] Nash J.: Continuity of solutions of parabolic and elliptic equations. Amer.J. Math. 80, 1958,pp.931-954.

[R-R] Rao M. M. and Ren Z. D.: Theory of Orlicz spaces, Monographs and Textbooks in Pure and Applied Mathematics, vol. 146, Marcel Dekker Inc., New York, 1991.

[Sc-S-V] Schilling R.L., Song R. and Vondraček Z.: Bernstein functions. Theory and applications. de Gruyter Studies in Mathematics, 37. Walter de Gruyter and Co., Berlin, 2010.

[S-S-V] Šikić H., Song R. and Vondraček Z.: Potential theory of geometric stable processes. Probab. Theory Related Fields 135 (2006), no. 4, 547-575.

[VSC] Varopoulos N.T., Saloff-Coste L. and Coulhon, T.: Analysis and Geometry on Groups. Cambridge University Press (1992).

[W1] Wang F.-Y.: Functional Inequalities for Dirichlet Operators with Powers. Chinese Sci. Tech. Online, 2007.

[W2] Wang F.-Y.: Functional inequalities for empty essential spectrum. J. Funct. Anal. 170 (2000), no. 1, 219-245.

[W3] Wang, F.-Y.: Functional inequalities for the decay of sub-Markov semigroups. Potential Analysis, 18 (2003), no. 1, 1-23.

[W4] Wang, F.-Y.: Functional Inequalities, Markov Processes and Spectral Theory. Science Press, Beijing, 2004. 